\title{
Erratum: Time reversal invariance violating and parity conserving effects in neutron-deuteron scattering [Phys. Rev. C 84, 025501 (2011)]
}

\author{
Young-Ho Song, Rimantas Lazauskas, and Vladimir Gudkov \\ (Received 24 February 2016; published 1 April 2016)
}

DOI: 10.1103/PhysRevC.93.049901

There was a technical mistake in calculations due to the singular behavior of Yukawa functions at short range. We corrected the integration algorithm. There were some typographical errors, which are corrected. The corrected tables are presented here.

The corrected equation (14) is as follows:

$$
\frac{f^{s t}}{p}=\frac{1}{2} \operatorname{Im} \frac{f_{+}+f_{-}}{p}=(-60.6+i 25.1) \mathrm{fm}^{2},
$$

giving the total cross section $\sigma_{\text {tot }}=\frac{4 \pi}{p} \operatorname{Im} f^{s t}(p)=3.15 \mathrm{~b}$.

The corrected equation (17) is

$$
\frac{1}{m_{N} C_{n}^{T P}} \frac{\Delta f^{T P}\left(\mu=m_{\pi}\right)}{p}=[(-0.03 \ldots 0.01)+i(-0.0004 \ldots 0.0013)] \mathrm{fm}^{2}
$$

The corrected equation (19) is

$$
\begin{aligned}
\Delta \sigma^{T P} & =10^{-6}\left[g_{h} \bar{g}_{h}(1.15)-g_{\rho} \bar{g}_{\rho}\left(4.22 \times 10^{-3}\right)\right] \mathrm{b}, \\
\frac{1}{N} \frac{d \phi^{T P}}{d z} & =10^{-3}\left[g_{h} \bar{g}_{h}(1.25)-g_{\rho} \bar{g}_{\rho}\left(5.76 \times 10^{-3}\right)\right] \mathrm{rad} \mathrm{\textrm {fm } ^ { 2 }} .
\end{aligned}
$$

All other equations, except for Eqs. (14), (17), and (19), and originally stated conclusions in the original paper are correct.

\begin{tabular}{|c|c|c|c|c|c|}
\hline$n$ & $\left\langle 2 \frac{3}{2}\left|v^{\frac{1}{2}}\right| 0 \frac{1}{2}\right\rangle / p^{2}$ & $\left\langle 1 \frac{3}{2}\left|v^{\frac{1}{2}}\right| 1 \frac{1}{2}\right\rangle / p^{2}$ & $\left\langle 2 \frac{3}{2}\left|v^{\frac{3}{2}}\right| 0 \frac{1}{2}\right\rangle / p^{2}$ & $\left\langle 1 \frac{3}{2}\left|v^{\frac{3}{2}}\right| 1 \frac{1}{2}\right\rangle / p^{2}$ & $\left\langle 2 \frac{5}{2}\left|v^{\frac{3}{2}}\right| 0 \frac{1}{2}\right\rangle / p^{2}$ \\
\hline 2 & $0.601 \times 10^{-4}$ & $0.567 \times 10^{-4}$ & $0.510 \times 10^{-5}$ & $-0.304 \times 10^{-4}$ & $0.346 \times 10^{-4}$ \\
\hline 4 & $-0.203 \times 10^{-4}$ & $-0.185 \times 10^{-4}$ & $-0.392 \times 10^{-5}$ & $0.954 \times 10^{-5}$ & $-0.952 \times 10^{-5}$ \\
\hline 5 & $-0.655 \times 10^{-4}$ & $-0.582 \times 10^{-4}$ & $-0.608 \times 10^{-5}$ & $0.305 \times 10^{-4}$ & $-0.283 \times 10^{-4}$ \\
\hline 6 & $-0.689 \times 10^{-5}$ & $0.124 \times 10^{-5}$ & $-0.414 \times 10^{-5}$ & $-0.573 \times 10^{-5}$ & $0.686 \times 10^{-5}$ \\
\hline 8 & $0.668 \times 10^{-4}$ & $0.563 \times 10^{-4}$ & $0.719 \times 10^{-5}$ & $-0.278 \times 10^{-4}$ & $0.252 \times 10^{-4}$ \\
\hline 9 & $0.388 \times 10^{-3}$ & $-0.262 \times 10^{-3}$ & $-0.136 \times 10^{-2}$ & $0.119 \times 10^{-3}$ & $-0.714 \times 10^{-3}$ \\
\hline 10 & $-0.114 \times 10^{-2}$ & $0.789 \times 10^{-3}$ & $0.411 \times 10^{-2}$ & $-0.359 \times 10^{-3}$ & $0.214 \times 10^{-2}$ \\
\hline 11 & $0.139 \times 10^{-6}$ & $0.837 \times 10^{-8}$ & $0.265 \times 10^{-8}$ & $0.166 \times 10^{-7}$ & $-0.268 \times 10^{-7}$ \\
\hline 12 & $-0.532 \times 10^{-5}$ & $-0.899 \times 10^{-6}$ & $-0.638 \times 10^{-5}$ & $-0.245 \times 10^{-8}$ & $0.446 \times 10^{-6}$ \\
\hline 13 & $-0.307 \times 10^{-4}$ & $0.104 \times 10^{-4}$ & $0.835 \times 10^{-4}$ & $-0.412 \times 10^{-5}$ & $0.407 \times 10^{-4}$ \\
\hline 18 & $0.346 \times 10^{-5}$ & $-0.242 \times 10^{-4}$ & $-0.257 \times 10^{-5}$ & $-0.442 \times 10^{-4}$ & $0.701 \times 10^{-6}$ \\
\hline
\end{tabular}

TABLE I. Representative contribution of each time reversal invariance violating and parity conserving (TVPC) potential term to the real part of the matrix element $\left(\frac{1}{C_{n}} \operatorname{Re} \frac{\left\langle\left(l^{\prime} j^{\prime}\right), J\left|V_{n}^{T P}\right|(l j), J\right\rangle}{p^{2}}\right)$. Results are presented using the $j j$-coupling scheme for wave functions obtained using the $A V 18+U I X$ interaction at $E_{\mathrm{cm}}=100 \mathrm{keV}$. For all operators a scalar function $\frac{m_{\rho}^{2}}{4 \pi} Y_{1}\left(m_{\rho} r\right)$ has been used. All data are in fm ${ }^{3}$. 
TABLE II. Same as in Table I but for the imaginary part of the matrix element $\left(\frac{1}{C_{n}} \operatorname{Im} \frac{\left\langle\left(l^{\prime} j^{\prime}\right), J\left|V_{n}^{T P}\right|(l j), J\right\rangle}{p^{2}}\right)$.

\begin{tabular}{|c|c|c|c|c|c|}
\hline$n$ & $\left\langle 2 \frac{3}{2}\left|v^{\frac{1}{2}}\right| 0 \frac{1}{2}\right\rangle / p^{2}$ & $\left\langle 1 \frac{3}{2}\left|v^{\frac{1}{2}}\right| 1 \frac{1}{2}\right\rangle / p^{2}$ & $\left\langle 2 \frac{3}{2}\left|v^{\frac{3}{2}}\right| 0 \frac{1}{2}\right\rangle / p^{2}$ & $\left\langle 1 \frac{3}{2}\left|v^{\frac{3}{2}}\right| 1 \frac{1}{2}\right\rangle / p^{2}$ & $\left\langle 2 \frac{5}{2}\left|v^{\frac{3}{2}}\right| 0 \frac{1}{2}\right\rangle / p^{2}$ \\
\hline 2 & $0.117 \times 10^{-2}$ & $-0.437 \times 10^{-2}$ & $0.139 \times 10^{-4}$ & $0.187 \times 10^{-2}$ & $0.947 \times 10^{-4}$ \\
\hline 4 & $-0.399 \times 10^{-3}$ & $0.143 \times 10^{-2}$ & $-0.107 \times 10^{-4}$ & $-0.587 \times 10^{-3}$ & $-0.260 \times 10^{-4}$ \\
\hline 5 & $-0.128 \times 10^{-2}$ & $0.449 \times 10^{-2}$ & $-0.166 \times 10^{-4}$ & $-0.187 \times 10^{-2}$ & $-0.773 \times 10^{-4}$ \\
\hline 6 & $-0.135 \times 10^{-3}$ & $-0.955 \times 10^{-4}$ & $-0.112 \times 10^{-4}$ & $0.353 \times 10^{-3}$ & $0.187 \times 10^{-4}$ \\
\hline 8 & $0.131 \times 10^{-2}$ & $-0.434 \times 10^{-2}$ & $0.196 \times 10^{-4}$ & $0.170 \times 10^{-2}$ & $0.688 \times 10^{-4}$ \\
\hline 9 & $0.761 \times 10^{-2}$ & $0.202 \times 10^{-1}$ & $-0.372 \times 10^{-2}$ & $-0.737 \times 10^{-2}$ & $-0.195 \times 10^{-2}$ \\
\hline 10 & $-0.225 \times 10^{-1}$ & $-0.608 \times 10^{-1}$ & $0.112 \times 10^{-1}$ & $0.221 \times 10^{-1}$ & $0.586 \times 10^{-2}$ \\
\hline 11 & $0.273 \times 10^{-5}$ & $-0.645 \times 10^{-6}$ & $0.719 \times 10^{-8}$ & $-0.102 \times 10^{-5}$ & $-0.732 \times 10^{-7}$ \\
\hline 12 & $-0.104 \times 10^{-3}$ & $0.693 \times 10^{-4}$ & $-0.174 \times 10^{-4}$ & $0.147 \times 10^{-6}$ & $0.120 \times 10^{-5}$ \\
\hline 13 & $-0.603 \times 10^{-3}$ & $-0.808 \times 10^{-3}$ & $0.228 \times 10^{-3}$ & $0.253 \times 10^{-3}$ & $0.111 \times 10^{-3}$ \\
\hline 18 & $0.678 \times 10^{-4}$ & $0.187 \times 10^{-2}$ & $-0.701 \times 10^{-5}$ & $0.272 \times 10^{-2}$ & $0.191 \times 10^{-5}$ \\
\hline
\end{tabular}

TABLE III. Difference of scattering amplitudes, $\frac{1}{C_{n}} \frac{\left(f_{n,+}-f_{n,-}\right)}{p}$ for TVPC potential from each operators and mass scales at $E_{\mathrm{cm}}=100 \mathrm{keV}$. Note that pion mass scale does not corresponds to physical meson exchange potential. All data are in femtometers.

\begin{tabular}{lrrr}
\hline \hline$n$ & $\frac{\Delta f^{\pi}}{p}$ & \multicolumn{1}{c}{$\frac{\Delta f^{\rho}}{p}$} & $\frac{\Delta f^{h_{1}}}{p}$ \\
\hline 1 & $0.16 \times 10^{-4}-i 0.85 \times 10^{-6}$ & $0.62 \times 10^{-6}-i 0.55 \times 10^{-7}$ & $-0.25 \times 10^{-6}-i 0.18 \times 10^{-7}$ \\
2 & $-0.45 \times 10^{-2}-i 0.39 \times 10^{-4}$ & $-0.18 \times 10^{-3}-i 0.36 \times 10^{-5}$ & $-0.53 \times 10^{-4}-i 0.10 \times 10^{-5}$ \\
3 & $0.37 \times 10^{-4}+i 0.26 \times 10^{-6}$ & $0.21 \times 10^{-5}+i 0.29 \times 10^{-7}$ & $0.74 \times 10^{-6}+i 0.10 \times 10^{-7}$ \\
4 & $0.15 \times 10^{-2}+i 0.14 \times 10^{-4}$ & $0.58 \times 10^{-4}+i 0.12 \times 10^{-5}$ & $0.17 \times 10^{-4}+i 0.35 \times 10^{-6}$ \\
5 & $0.49 \times 10^{-2}+i 0.16 \times 10^{-3}$ & $0.18 \times 10^{-3}+i 0.41 \times 10^{-5}$ & $0.53 \times 10^{-4}+i 0.11 \times 10^{-5}$ \\
6 & $-0.24 \times 10^{-2}+i 0.23 \times 10^{-4}$ & $-0.19 \times 10^{-4}+i 0.32 \times 10^{-6}$ & $-0.21 \times 10^{-5}+i 0.68 \times 10^{-7}$ \\
7 & $-0.14 \times 10^{-3}-i 0.14 \times 10^{-5}$ & $-0.66 \times 10^{-5}-i 0.91 \times 10^{-7}$ & $-0.23 \times 10^{-5}-i 0.31 \times 10^{-7}$ \\
8 & $-0.40 \times 10^{-2}-i 0.16 \times 10^{-3}$ & $-0.17 \times 10^{-3}-i 0.42 \times 10^{-5}$ & $-0.51 \times 10^{-4}-i 0.11 \times 10^{-5}$ \\
9 & $0.42 \times 10^{-1}-i 0.20 \times 10^{-2}$ & $0.14 \times 10^{-2}-i 0.73 \times 10^{-4}$ & $0.44 \times 10^{-3}-i 0.22 \times 10^{-4}$ \\
10 & $-0.12 \times 10^{+0}+i 0.63 \times 10^{-2}$ & $-0.43 \times 10^{-2}+i 0.22 \times 10^{-3}$ & $-0.13 \times 10^{-2}+i 0.68 \times 10^{-4}$ \\
11 & $0.61 \times 10^{-5}-i 0.91 \times 10^{-6}$ & $0.93 \times 10^{-7}-i 0.73 \times 10^{-8}$ & $0.62 \times 10^{-8}-i 0.11 \times 10^{-8}$ \\
12 & $-0.58 \times 10^{-3}-i 0.80 \times 10^{-4}$ & $-0.96 \times 10^{-6}+i 0.11 \times 10^{-6}$ & $-0.15 \times 10^{-7}+i 0.22 \times 10^{-7}$ \\
13 & $0.95 \times 10^{-3}-i 0.41 \times 10^{-4}$ & $-0.71 \times 10^{-4}+i 0.49 \times 10^{-5}$ & $-0.25 \times 10^{-4}+i 0.17 \times 10^{-5}$ \\
14 & $-0.23 \times 10^{-2}+i 0.93 \times 10^{-4}$ & $0.22 \times 10^{-3}-i 0.15 \times 10^{-4}$ & $0.75 \times 10^{-4}-i 0.50 \times 10^{-5}$ \\
15 & $-0.52 \times 10^{-6}-i 0.96 \times 10^{-7}$ & $0.16 \times 10^{-7}-i 0.61 \times 10^{-9}$ & $0.43 \times 10^{-8}-i 0.38 \times 10^{-10}$ \\
16 & $-0.18 \times 10^{-3}+i 0.10 \times 10^{-4}$ & $-0.46 \times 10^{-6}+i 0.28 \times 10^{-7}$ & $-0.28 \times 10^{-7}+i 0.24 \times 10^{-8}$ \\
17 & $0.17 \times 10^{-3}+i 0.16 \times 10^{-4}$ & $0.76 \times 10^{-5}+i 0.64 \times 10^{-8}$ & $0.22 \times 10^{-5}-i 0.38 \times 10^{-8}$ \\
18 & $0.27 \times 10^{-3}-i 0.22 \times 10^{-4}$ & $0.87 \times 10^{-5}-i 0.32 \times 10^{-6}$ & $0.22 \times 10^{-5}-i 0.76 \times 10^{-7}$ \\
\hline \hline
\end{tabular}

